\title{
Data Communication Through the Atmospheric Burst Error Channel
}

\author{
N. S. JAYANT
}

REFERENCE: Jayant, N. S.: DATA COMMUNICATION THROUGH THE ATMOSPHERIC BURST ERROR CHANNEL, Indian Institute of Science, Bangalore, India. Rec'd 10/31/66; revised $1 / 24 / 67$. Paper 19TP67-932. IEEE TRANS. ON COMMUNICATION TECHNOLOGY, 15-3, June 1967, pp. 383-389.

\begin{abstract}
Simple and easily utilized techniques are suggested for reliable transmission of binary data in the presence of atmospheric noise bursts. The techniques utilize the time statistics of the noise bursts, now known to be $\log$-normal, and operate on one-way channels. Two modes of transmission are distinguished. The first mode involves double or triple transmission of an error detection code word. The second mode of transmission is based on burst error correction, provided either by a majority count principle or by the recurrent codes of Hagelbarger. Numerical calculations indicate the superior performance of the error detection techniques.
\end{abstract}

KEYWORDS: Analysis, Atmospherics, Coding, Communication Theory, Data Transmission Systems, Digital Signals, Errors, High Frequency.

\section{InTRODUCTION}

$A^{1}$ TMOSPHERIC noise constitutes an important source of interference to digital communication over a wide range of frequencies. In certain geographical locations, such noise is predominantly in the form of distinct bursts superposed on a background of low level interference. ${ }^{[1],[2]}$ The average duration of an atmospheric noise burst is of the order of 0.5 second, and is much greater than the practically allowable duration of a message element. The noise bursts thus tend to introduce corresponding clusters of errors in the decoding of data transmissions.

The time statistics of such error bursts--in particular, the ratio of the average duration of an error burst to the mean value of the burst repetition period - often preclude a profitable utilization of available techniques of error control. Modified schemes are therefore suggested in this paper and are evaluated using available noise burst data. Specifically, two modes of transmission are distinguished as they involve error detection or error correction at the receiver. The recurrent codes of Hagelbarger ${ }^{[3]}$ are included for discussion under the error correction mode.

The analysis of the proposed techniques will assume a zero signal-to-noise ratio throughout the duration of a noise burst. Numerical results that will be presented on the failure rates in these techniques will therefore be higher than those to be expected in practical systems. But the results will serve to illustrate relative performances of different transmission schemes as used over burst error channels.

The subject matter of the paper is arranged as follows. The nature of the problem is first briefly assessed in Sec- tion II. The short term time characteristics of atmospheric noise bursts are discussed in Section III. The proposed techniques are described in Section IV, which includes analyses of performance. Available noise burst data are summarized in Section $V$. These are used to evaluate techniques in Section VI and VII. Mathematical derivations are furnished in the Appendixes.

\begin{tabular}{|c|c|}
\hline \multicolumn{2}{|r|}{ NOTATION } \\
\hline$p, q, r$ & $\begin{array}{l}\text { Random variables denoting the duration of a } \\
\text { noise burst, the time interval between suc- } \\
\text { cessive bursts, and the burst repetition period }\end{array}$ \\
\hline $\bar{\alpha}, \alpha_{m}, \overline{\alpha^{2}}$ & Mean, median, and mean square values of $\alpha$ \\
\hline$\alpha$ & $p, q$, or $r$ \\
\hline$\sigma_{\alpha}$ & Standard deviation of $\ln \alpha$ \\
\hline$N_{0}$ & Number of noise bursts received per minute \\
\hline$\tau$ & Duration of a message element \\
\hline$A, B$ & $\begin{array}{l}\text { Refer to the error detection and error correc- } \\
\text { tion modes of transmission, respectively }\end{array}$ \\
\hline $\begin{array}{l}A 1, A 2 \\
\quad(B 1, B 2)\end{array}$ & Refer to two techniques in mode $A$ (and $B$ ) \\
\hline$D_{1}\left(d_{1}\right)$ & Optimum message block length in $A 1$ (and $B 1$ ) \\
\hline$D_{2}$ & Optimum message block length in $A 2$ \\
\hline$d_{2}$ & $\begin{array}{l}\text { Optimum value of recurrent code parameter in } \\
B 2\end{array}$ \\
\hline$\lambda_{1}\left(\lambda_{2}\right)$ & $\begin{array}{l}\text { Redundancy of error detecting code in } A 1 \\
\text { (and } A 2 \text { ) }\end{array}$ \\
\hline$F_{S}$ & $\begin{array}{l}\text { Upper bound on the minimum failure rate in } \\
\text { scheme } S\end{array}$ \\
\hline$S$ & $A 1, A 2, B 1$, and $B 2$. \\
\hline
\end{tabular}
$\Psi\left[ \pm x^{(\alpha)} \pm y\right] \equiv \frac{1}{\sqrt{2 \pi}} \int_{ \pm \frac{1}{\sigma \alpha} \ln (x / \alpha m) \pm y}^{\infty} \exp \left(-u^{2} / 2\right) d u$.

\section{The Problem}

Previous work on transmissions over burst error channels has largely referred to the problem of telephone line bursts, as in Reiffen et al., ${ }^{[4]}$ and, more recently, to the Gilbert model. ${ }^{\left[{ }^{[}\right]}$In systems permitting two-way communication, the possibility of efficient data communication, through the use of error detection at the receiver and requested retransmissions, has been generally demonstrated. Specifically, Bose-Chaudhari codes have been employed for error detection, ${ }^{[4]}$ and, on the theoretical side, the problem of designing message block length with a view to maximizing the information rate has been investigated. ${ }^{[5]}$ 
The problem of data communication in the presence of atmospheric noise bursts is generally more difficult. This is because the ratio of the mean duration $\bar{p}$ of such bursts to the mean burst repetition period. $\bar{r}$ is in the range 0.1 and 0.2 (Section VI), and such conditions render the simple ARQ (automatic request for repeat) technique inefficient. The probability of a repeat request will be of the order of $\bar{p} / \bar{r}$ for short messages (message length $\ll \bar{r}$ ) and much more for longer messages. Furthermore, in the latter case, which is of practical importance, the probability of a re-repeat request will also be considerable. Under these circumstances, it may be much more convenient (although perhaps less efficient from the point of view of overall information rate) to arrange for a fixed number of transmissions $R$ of an error detection code at the source, and to design the length and spacing of such transmissions so as to ensure a high probability that at least one of them is uninfluenced by noise bursts and is correctly: received. Such a prosedure will minimize the problems of storage at the transmitter, and will obviate the need for a feedback link. Similar considerations apply when messages are encoded for burst error correction. In either case, the time statistics of noise bursts will determine the design of the transmissions.

Transmission techniques ${ }^{[6],[7]}$ which exploit the time statistics of noise bursts more effectively than the schemes of this paper, have been suggested. The codes of this paper are really not optimal for the noise environment considered. But they furnish techniques which do not require elaborate experimental verification, and can be used directly on practical circuits.

\section{Short Term Time Characteristics of Atmospheric NoIsE BuRsts}

The duration of an atmospheric noise burst $p$ and the repetition period of such bursts $r$ have been found to be log-normally distributed. ${ }^{[1],[2],[8]}$ The log-normal distribution of $\alpha$ is described by the following relations.

The density function of $\alpha$ is given by

$$
f(\alpha)=\frac{1}{\sqrt{2 \pi} \cdot \sigma_{\alpha} \cdot \alpha} \exp \left[-\left\{\frac{\ln \left(\alpha / \alpha_{m}\right)}{\sqrt{2} \cdot \sigma_{\alpha}}\right\}^{2}\right]
$$

where $\alpha_{m}$ is the median value of $\alpha$ and $\sigma_{\alpha}$ is the standard deviation of $\ln \alpha$. Furthermore, the mean value of $\alpha$ is

$$
\bar{\alpha}=\alpha_{m} \cdot \exp \left(\sigma_{\alpha}{ }^{2} / 2\right)
$$

and the mean square value is

$$
\overline{\alpha^{2}}=\alpha_{m}{ }^{2} \cdot \exp \left(2 \sigma_{\alpha}^{2}\right) .
$$

The log-normal distribution can be reduced to the normal distribution, with a mean of zero and a standard deviation of unity, by the transformation

$$
\alpha \rightarrow \alpha_{m} \cdot \exp \left(\sigma_{\alpha} \cdot u\right) .
$$

Therefore, we may write

$$
\begin{array}{r}
\operatorname{Prob}(\alpha>A)=\frac{1}{\sqrt{2 \pi}} \int_{1 / \sigma \alpha \ln (A / \alpha m)}^{\infty} \exp \left(-u^{2} / 2\right) d u \\
=\Psi\left[A^{(\alpha)}\right] .
\end{array}
$$

Experimental investigations at tropical latitudes indicate that the short term distributions of $p$ and $r$ are sta.tistically independent. It can then be shown (Appendix I) that the distribution of the time interval $q$ between successive bursts is also log-normal, to an approximation, and has the moments

$$
\begin{aligned}
& \bar{q}=\bar{r}-\bar{p} \\
& \sigma_{q}{ }^{2}=\ln \left[1+\frac{(\bar{r})^{2}\left(\exp \sigma_{\tau}{ }^{2}-1\right)-(\bar{p})^{2}\left(\exp \sigma_{p}{ }^{2}-1\right)}{(\bar{q})^{2}}\right]
\end{aligned}
$$

\section{The Proposed Techniques}

In what follows, it is assumed that $\tau \ll \bar{p} \ll \bar{q}$, where $\tau$ is the duration of a message element and $\bar{p}$ and $\bar{q}$ are the mean values of the duration of a noise burst and the time interval between successive bursts.-It is futther assumed that a message element which is not influenced by a noise burst, i.e., an element which falls outside the duration of the burst, in time, is correctly decoded, with probability one. The effect of background (Gaussian) interference on binary transmissions can be rendered negligible by providing a signal-to-background-noise ratio of about $15 \mathrm{~dB}^{\left[{ }^{[9]}\right.}$ A message element, part or all of which falls within the duration of a noise burst, is considered, on the other hand, to be wrongly decoded with probability one. ${ }^{1}$ This assumption leads to the establishment of upper bounds on the failure rates of the techniques to be considered. The bounds will apply irrespective of the modem employed.

\section{A. Techniques Involving Error Detection}

Message blocks in this transmission mode are in the form of error detecting codes. Bose-Chaudhari codes, for instance, can be used for reliable error detection over burst error channels. ${ }^{[4]}$ An $(m, t)$ Bose-Chaudhari code is of length $2^{m}-1$, contains $m t$ redundant elements, and is $t$ error correcting. In the event of more than $t$ errors, the code still detects at least one error with a probability of the order of $1-2^{-m t}$. The probability of undetected errors can therefore be reduced to a negligible value by the provision of sufficient redundancy. Since the techniques to be described are based primarily on this error detecting property of the codes, their error correcting capabilities will not be considered in the analysis. A message block is assumed to be correctly received only. if the corresponding transmission falls entirely in the time interval between two adjacent noise bursts.

It is proposed to improve the reliability of such communications by transmitting every error detecting code a fixed number of times $R$ so as to ensure a high probability that at least one of the $R$ transmissions falls in an interval between adjacent noise bursts. This probability can be arbitrarily increased by increasing $R$. The discussion in

\footnotetext{
1 The density of errors within a burst may be much smaller than unity on practical circuits. Its effect, however, on the failure rates of the proposed schemes will be seen to be small if $r \ll \bar{p}$ (also see Section VII, paragraph 3 ).
} 
this paper is restricted, however, to the cases of $R=3$ and 2 . These are the cases most likely to be of practical significance. Furthermore, they typify two different classes of techniques in the error detection mode, viz., techniques involving consecutive retransmissions and those employing spaced retransmissions of a message block.

1) A Technique Involving Three Transmissions: The error detection codes in this technique are of length $D_{1}$, and are transmitted consecutively thrice. $D_{1}$ is designed to maximize the probability that at least one of the three transmissions is uninfluenced by noise bursts.

It can be seen that a sufficient condition for the noncorruption of at least one of the three consecutive transmissions is the inequality $q / 2>D_{1}>p$. The time functions $p$ and $q$ are, however, random variables. The design of the code length $D_{1}$ should, therefore, be optimized in terms of the statistics of $p$ and $q$ to ensure a maximum reliability of communication. The optimization procedure is indicated in what follows.

If the code length in the scheme is, say, $s_{1}$, a transmission failure can occur due to a) an overlong noise burst $\left(p>s_{1}\right)$ and/or b) an overshort interval between successive bursts $\left(q<2 s_{1}\right)$, provided these are so positioned, in time, as to influence all the three transmissions of a code. (For instance, an overlong burst corrupting both the first and second transmissions of a code can still leave the third unaffected, while, if centered on the second transmission, the burst may extend sufficiently into the first and third blocks to corrupt all the three transmissions of a code. Similar considerations apply for the corruption of the transmissions due to an overshort interval between successive bursts.) On the basis of the previous discussion, an upper bound $F\left(s_{1}\right)$ is derived on the transmission failure rate in the scheme (Appendix II) and reads

$$
\begin{aligned}
F\left(s_{1}\right)=\frac{1}{\bar{r}}\left\{\bar{p} \cdot \Psi\left[s_{1}{ }^{(p)}-\sigma_{p}\right]-s_{1} \cdot \Psi\left[s_{1}{ }^{(p)}\right]+\right. \\
\left.4 s_{1} \cdot \Psi\left[-2 s_{1}{ }^{(q)}\right]-2 \bar{q} \cdot \Psi\left[-s_{1}{ }^{(q)}+\sigma_{q}\right]\right\}
\end{aligned}
$$

where

$\Psi\left[ \pm x^{(\alpha)} \pm y\right]=\frac{1}{\sqrt{2 \pi}} \int_{ \pm 1 / \sigma \alpha \ln \left(x / \alpha_{m}\right) \pm y}^{\infty} \exp \left(-u^{2} / 2\right) d u$.

The optimum code length $D_{1}$ is the value of $s_{1}$ which minimizes $F\left(s_{1}\right)$. Correspondingly, the minimum failure rate in the scheme has the upper bound

$$
F_{A 1}=F\left(D_{1}\right) \text {. }
$$

Both $D_{1}$ and $F_{A 1}$ can be computed numerically from a knowledge of the time statistics of the noise bursts.

The redundancy involved in the technique is $0.33(2+$ $\lambda_{1}$ ) where $\lambda_{1}$ is the redundancy of the error detecting code employed.

2) A Technique Involving Two Transmissions: The middle block in the three transmissions of a code in the previous technique $\mathrm{Al}$ is rather redundant. It is possible to work with two spaced transmissions of a code rather than three consecutive transmissions, and still ensure a high probability that one of the transmissions is uninfluenced by noise bursts, and is correctly received. The performance of such a technique will be analyzed in what follows.

Specifically, the codes in this scheme will be of length $D_{2}$ and are transmitted twice, with a spacing also equal to $D_{2}$. (Such a spacing can accommodate one of the two transmissions of another message block.) A sufficient condition for the noncorruption of at least one of the two transmissions of a code is the inequality $q / 3>D_{2}>p$. As in the previous scheme, the design of the code length $D_{2}$ is based on the statisties of $p$ and $q$.

If the code length used is, say, $s_{2}$, a transmission failure can occur due to a) an overlong burst $\left(p>s_{2}\right)$ and/or b) an overshort interval between successive bursts $(q<$ $3 s_{2}$ ), provided these are so positioned, in time, as to influence both the transmissions of a code. An upper bound $F^{\prime}\left(s_{2}\right)$ can be derived on this basis for the probability of transmission failure in the scheme. From Appendix II

$$
\begin{aligned}
F^{\prime}\left(s_{2}\right)=\frac{2}{3 \bar{r}}\left\{\bar{p} \cdot \Psi\left[s_{2}{ }^{(p)}-\sigma_{p}\right]-s_{2} \cdot \Psi\left[s_{2}{ }^{(p)}\right]+\right. \\
\\
\left.\quad 3 s_{2} \cdot \Psi\left[-3 s_{2}{ }^{(q)}\right]-\bar{q} \cdot \Psi\left[-3 s_{2}{ }^{(q)}+\sigma_{q}\right]\right\} .
\end{aligned}
$$

The optimum code length $D_{2}$ is the value of $s_{2}$ which minimizes $F^{\prime}\left(s_{2}\right)$. Correspondingly, the minimum transmission failure rate in the technique has the upper bound

$$
F_{A 2}=F^{\prime}\left(D_{2}\right) \text {. }
$$

The scheme involves a redundancy of $0.5\left(1+\lambda_{2}\right)$ where $\lambda_{2}$ is the redundancy of the error detecting code employed.

\section{B. Techniques Involving Error Correction}

The techniques to be described are burst error correcting. They are inherently less reliable than the schemes of Section IV-A, but have the advantage of much simpler implementation.

1) A Majority Count Technique: Message blocks in this scheme are original, uncoded sequences of length $d_{1}$, where $d_{1}$ is designed as the value of $a_{1}$ which minimizes [Prob $\left(p>a_{1}\right)+$ Prob $\left.\left(q<2 a_{1}\right)\right] ; p$ and $q$ refer to the duration of a noise burst and the time interval between successive bursts. From Appendix III

$$
d_{1}=\left[p_{m}{ }^{\sigma_{q}} \cdot\left(q_{m} / 2\right)^{\sigma_{p}}\right]^{1 /\left(\sigma_{p}+\sigma_{q}\right)} .
$$

Every message block is transmitted consecutively thrice. The choice of the bit retransmission period $d_{1}$ ensures, at the receiver, for every message element $Q_{D}$ (at time $T$ ) corrupted by a noise burst, two corresponding duplicates $Q_{E}$ and $Q_{F}$ (at times $T \pm d_{1}, T \pm 2 d_{1}$ ) which have a high probability of being uncorrupted. The decoding is therefore carried out in two stages. In the first, the incoming data stream is serially decoded into Marks (M) and Spaces (S). In the second stage, decisions are taken on 
a message element $Q$ on the basis of: a majority count over the corresponding triplicate $Q_{D}, Q_{E}$, and $Q_{F}$. That is, $q$ is announced as M (or $\mathrm{S}$ ) accordic gly as two or more of $Q_{D}$, $Q_{E}$, and $Q_{F}$ are decoded as $\mathrm{M}$ (cr $\mathrm{S}$ ). This rule can lead to erroneous decisions due to a) an overlong noise burst $\left(p>d_{1}\right)$ and/or b) an overshort interval between successive bursts $\left(q<2 d_{1}\right)$, each of which can result in the corruption of more than one element in one or more triplicates. On this basis, the following upper bound $F_{B 1}$ is derived (see Appendix IV) for the minimum transmission failure rate in the scheme.

$$
F_{B 1}=\frac{6 d_{1}}{\bar{r}} \cdot \Psi\left[d_{1}^{(p)}\right]
$$

2) The Use of Recurrent Codes: The technique B1 described in the previous subsection was clearly burst error correcting. A closely related procedure happens to be the encoding of messages into the 50 percent redundancy, $(2,1)$ recurrent codes of Hagelbarger. [3] The performance of these codes, as used over the atmospheric noise burst channel, is now analyzed.

The recurrent codes previously mentioned can be designed to correct error bursts of length equal to or less than a number, say, $d_{2} / \tau$ of bits, conditional to the provision of a minimum error-free guard space of $3 d_{2} / \tau+1$ bits between successive bursts. The error bursts at the output of a decoder have time statistics corresponding essentially to those of the noise bursts, viz., the statistics of $p$ and $q$. The parameter $d_{2}$ of the recurrent codes is therefore chosen as the value of $a_{2}$ which minimizes [Prob $\left(p>a_{2}\right)+$ Prob $\left(q<3 a_{2}\right)$ ]. It has been assumed that $3 d_{2} / \tau \gg 1$; this will be true if $\tau \ll \bar{p}$ and $\bar{q}$. From Appendix III

$$
d_{2}=\left[p_{m}{ }^{\sigma_{p}} \cdot\left(q_{m} / 3\right)^{\sigma_{q}}\right]^{1 /\left(\sigma_{p}+\sigma_{q}\right)} \text {. }
$$

The mechanism of failure in this scheme is similar to that in the majority count technique. Decoding errors will be the result of a) overlong noise bursts $\left(p>d_{2}\right)$ and/or b) overshort intervals between successive bursts $\left(q<3 d_{2}\right)$. The minimum failure rate has an upper bound $F_{B 2}$ which reads (see Appendix IV)

$$
F_{B 2}=\frac{4 d_{2}}{\bar{r}} \cdot\left[\left[d_{2}{ }^{(p)}\right]\right.
$$

\section{Noise Burst Data}

Available data on the time statistics of noise bursts are summarized in this section, and will be utilized to evaluate the proposed techniques in Sections VI and VII. Data on the short term distributions of $p$ and $r$ and on the long term variations of the burst occurrence rate $N_{0}$, are available for tropical locations. ${ }^{[1],[2],[8]}$ The following values are typical: $\bar{p}=500 \mathrm{~ms}, \sigma_{p}=0.63, \sigma_{r}=0.44$. Data on $\sigma_{p}$ and $\sigma_{r}$ are in log-units in the literature and have been converted here into the natural units of Section III. Results show that $N_{0}$ varies with season and the hour of day. It has been stated that $N_{0}$ can be taken as 20 per minute, correct to a factor of two either way, whenever noise bursts occupy more than 10 percent of the time. But, particularly at $\mathrm{HF}$, values of $N_{0}$ much smaller than 10 per minute have also been reported. Taking an overall view, $N_{0}$ will be 20 per minute or less for a considerable portion of the time.

\section{Illustra'itive Calculations}

The results of Section IV are now illustrated by numerical calculations on the transmission failure rates. Discussions in Sections III and V justify the following assumptions:

$$
\begin{gathered}
5<q_{m} / p_{m}<10 \\
\sigma_{p}=\sigma_{q}=0.6 .
\end{gathered}
$$

The cases $q_{m} / p_{m}<5$ and $q_{m} / p_{m}>10$ will be discussed in Section VII. Further, to illustrate the dependence of the failure rates on $\sigma_{p}$ and $\sigma_{q}$, the following pair of values is also included for the calculations:

$$
\sigma_{p}=\sigma_{q}=0.3
$$

Upper bounds on the minimum transmission failure rates in the schemes of Section IV, calculated by using (17), (18), and (19), are given in Table I.

The minima of the transmission failure rates, which are functions of $s_{1}, s_{2}, a_{1}$, and $a_{2}$ (see Section IV), are found to be very broad, as calculated using (17), (18), and (19). Rounded values of the corresponding optimum message block lengths $D_{1}, D_{2}$, and $d_{1}$, and of $d_{2}$, are given in Table II as multiples of $p_{m}$.

\section{TABLE I}

\begin{tabular}{|c|c|c|c|c|}
\hline & $\begin{array}{c}q_{m} \\
\sigma_{p}= \\
\sigma_{q}= \\
0.3\end{array}$ & $\begin{array}{c}5 p_{m} \\
\sigma_{p}= \\
\sigma_{g}= \\
0.6\end{array}$ & $\begin{array}{c}q_{m}= \\
\sigma_{p}= \\
\sigma_{q}= \\
0.3\end{array}$ & $\begin{array}{c}10 p_{m} \\
\sigma_{p}= \\
\sigma_{q}= \\
0.6\end{array}$ \\
\hline $\begin{array}{l}D_{1} \\
D_{2} \\
d_{1} \\
d_{2}\end{array}$ & $\begin{array}{l}1.5 \\
1.1 \\
1.6 \\
1.3\end{array}$ & $\begin{array}{l}1.1 \\
1.0 \\
1.6 \\
1.3\end{array}$ & $\begin{array}{l}2.2 \\
1.4 \\
2.2 \\
1.8\end{array}$ & $\begin{array}{l}1.8 \\
1.4 \\
2.2 \\
1.8\end{array}$ \\
\hline
\end{tabular}

Upper Bounds on the Minimum Failure Rates of Differen't Technigues (Bounds Are Parts in 100)

\begin{tabular}{ccccc} 
& $q_{m}=5 p_{m}$ & $q_{m}=10 p_{m}$ \\
& $\sigma_{p}=$ & $\sigma_{p}=$ & $\sigma_{p}=$ & $\sigma_{p}=$ \\
Techniques as & $\sigma_{q}=$ & $\sigma_{q}=$ & $\sigma_{q}=$ & $\sigma_{q}=$ \\
in Section IV & 0.3. & 0.6 & 0.3 & 0.6 \\
\hline A1 & 1 & 5 & 0.05 & 1 \\
A2 & 1.5 & 5 & 0.2 & 1 \\
B1 & 10.0 & 30 & 0.5 & 10 \\
B2 & 15.0 & 20 & 1.5 & 10
\end{tabular}

TABLE II

Values of $D_{1}, D_{2}, d_{1}$ and $d_{3}$, as Multiples of $p_{m}$ 
It is useful to add that, for the trivial case of $\sigma_{p}=$ $\sigma_{q}=0$, the values of $d_{1}$ and $D_{1}$ can range from $p_{m}$ to $q_{m} / 2$ and those of $d_{2}$ and $D_{2}$, from $p_{m}$ to $q_{m} / 3$.

\section{Discussion}

As mentioned before, the analyses of the proposed techniques were based on the assumption of zero signal-tonoise ratio throughout the duration of a noise burst. In practical systems, provision of large signal power can reduce the failure rates in these techniques to values arbitrarily lower than the bounds in Table I. A possible method of refining the analysis for such cases would consist in working with the time statisties of the noise bursts as measured at a chopping level corresponding to the signal amplitude. Experimental results indicate that such statistics are also log-normal. ${ }^{10]}$

Certain general conclusions can be drawn from Table I. The transmission failure rates are increasing functions of $p_{m} / q_{m}$ and of $\sigma_{p}$ and $\sigma_{q}$, and are always less in the error detection mode $\mathrm{A}$ than in the error correction mode $\mathrm{B}$. Within each mode, at small values of $\sigma_{p}$ and $\sigma_{\psi}$, the use of increased redundancy (as in A1 and B1) can be traded for a decrease in failure rate. However, at large values of $\sigma_{p}$ and $\sigma_{q}$ (these are the cases of practical significance), the techniques $\mathrm{A} 2$ and $\mathrm{B} 2$ tend to perform better than $\mathrm{A} 1$ and B1.

The error correction techniques of Section IV-B have a limited application in view of their poor reliability at small values of $q_{m} / p_{m}$. However, if their use is sought in view of their simplicity, it will be imperative to work not only with large signal power, but, also, with as large a value as is practicable for the message element duration $\tau$. It can be shown that this will reduce the failure rates derived in the analysis by a factor of the order of $P_{e}{ }^{2} \bar{p} / \tau$, where $P_{e}$ is the probability of bit error conditional to the occurrence of a noise burst.

The range $5<q_{m} / p_{m}<10$ (see Section VI) is significant. While the reliability of the proposed techniques is an increasing function of $q_{m} / p_{m}$, they will really be suboptimum at very large values of the ratio (say, greater than 10), because, in such conditions, an ARQ logic could provide a better information rate. On the other hand, at values of $q_{m} / p_{m}$ smaller than about five, the failure rates in the techniques will be too large to justify their application. In fact, as far as the author is aware, no schemes are available for reliable transmission of data over channels characterized by such small values of $q_{m} / p_{m}$, except by the provision of enormous signal energy and/or redundancy, or, alternatively, by an equivalent refinement of receiver structure. The latter tendency is exemplified in the time diversity system of Lyons, ${ }^{[6]}$ and by the scheme proposed by Jayant $^{[7]}$ where the use of a nonlinear null-zone receiver converts the atmospheric burst error channel into an erasure channel with an erasure probability that can, in principle, be held arbitrarily low.

The investigations reported in this paper were largely motivated by noise data available for tropical locations. It is quite possible, however, that the analysis of Section
IV is also applicable to data systems at other geographical locations.

\section{APPENDIX I}

The distribution of $q=r-p$

The functions $r$ and $p$ are log-normally distributed, and statistically independent. Numerical convolution indicates that the distribution of $q$ is also log-normal to a good approximation. Assuming such a distribution for $q$, the constants $\bar{q}$ and $\sigma_{q}$ can be determined analytically on the basis of the following equivalence of means and central moments: ${ }^{[11]}$

$$
\begin{gathered}
\bar{q}=\bar{r}-\bar{p} \\
\bar{q}^{2}-(\bar{q})^{2}=\left[\bar{r}^{2}-(\bar{r})^{2}\right]-\left[\bar{p}^{2}-(\bar{p})^{2}\right] .
\end{gathered}
$$

Such an equivalence provides a good fit to the exact distribution of $q$ in the middle range, say, $\bar{q} / \sqrt{10}<q<$ $\sqrt{10} \bar{q}$, of the distribution. The analysis included in this paper is essentially concerned with the lower part of this range. From practical considerations, values of $q$ less than about $\bar{q} / \sqrt{10}$ will be of little interest. Through the results of Section III, (21) simplifies to the form

$\sigma_{q}{ }^{2}=\ln \left[1+\frac{(\bar{r})^{2}\left(\exp \sigma_{r}{ }^{2}-1\right)-(\bar{p})^{2}\left(\exp \sigma_{p}{ }^{2}-1\right)}{(\bar{q})^{2}}\right]$

Equations (20) and (22) describe the log-normal fit to the distribution of $q$.

\section{Appendix II}

In what follows, it is assumed that the positioning of a noise burst (or a burst-free interval) relative to the message transmissions follows a rectangular distribution; that is, if the beginning of a burst (or a burst-free interval) is displaced by a time $l$ (or $m$ ) from the starting of the first of $R$ transmissions of a message block $(R=2$ or 3 ), Prob $(l<n)=\operatorname{Prob}(m<n)=n / 3 s ; s$ is the code length and $3 s$ is the total transmission time of a message in the schemes of Section IV-A.

\section{Derivation of $F\left(s_{1}\right)$}

It is necessary to derive an expression for the failure rate in scheme A1 while working with a code length $s_{1}$. A failure can occur in the scheme if all the three transmissions of a message block are influenced by a noise burst or bursts.

Consider an overlong burst of duration $p>s_{1}$. Such a burst can cause a transmission failure if it is centered on the middle block (of a set of three transmissions) and extends in to the first and third blocks. This has a probability equal to $\left(p-s_{1}\right) / 3 s_{1}$ [Fig. 1(a)] if $p<4 s_{1}$, and equal to unity if $p>4 s_{1}$. Therefore, averaging over the distribution of $p$, the probability of a noise burst causing a transmission failure has an upper bound of

$$
\int_{s_{1}}^{\infty} \frac{p-s_{\mathbf{1}}}{3 s_{1}} \cdot f(p) d p
$$




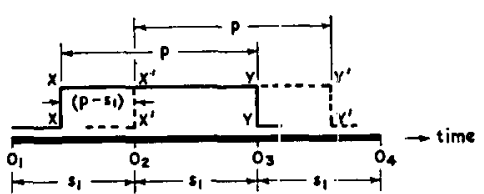

(a)

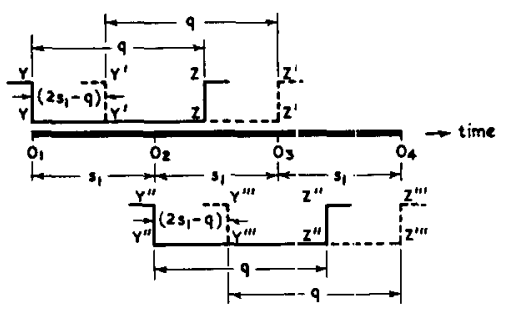

(b)

Fig. 1. Mechanism of transmission failure in A1. (a) $\mathrm{XX}^{\prime} /$ $\mathrm{O}_{1} \mathrm{O}_{4}$ is the fraction of time for which the burst $\mathrm{X} Y$ influences all the three transmissions; $\mathrm{O}_{1} \mathrm{O}_{2}, \mathrm{Q}_{2} \mathrm{O}_{3}$, and $\mathrm{O}_{3} \mathrm{O}_{4 \cdot}$. (b) $\left(Y Y^{\prime}+\right.$ $\left.\mathrm{Y}^{\prime \prime} \mathrm{Y}^{\prime \prime \prime}\right) / \mathrm{O}_{1} \mathrm{O}_{4}$ is the fraction of time for which adjacent bursts, separated by the interval $\mathrm{Y} Z$, influence all the three transmission $\mathrm{O}_{2} \mathrm{O}_{2}, \mathrm{O}_{2} \mathrm{O}_{3}$, and $\mathrm{O}_{3} \mathrm{O}_{4}$.

Similarly, the probability of transmission failure caused by an overshort interval between adjacent bursts [Fig. 1(b) ] has an upper bound of

$$
\int_{0}^{2 s_{1}} \frac{2\left(2 s_{1}-q\right)}{3 s_{1}} \cdot f(q) d q
$$

where it has been assumed that $p<s_{1}$.

Consider $N$ transmissions under the scheme, extending over a time $3 s_{1} N$. The number of noise bursts and that of burst-free intervals, occurring in this period, tends to $3 s_{1} N / \bar{r}$ for large $N$ ( $\bar{r}$ is the burst repetition period). Each of these bursts and intervals can be associated with a probability of causing a transmission failure, bounded by the integrals (23) and (24), respectively. Therefore, the overall failure rate in the scheme has an upper bound

$$
\frac{3 s_{1} N}{\bar{r}}\left[\int_{s_{1}}^{\infty} \frac{p-s_{1}}{3 s_{1}} \cdot f(p) d p+\int_{0}^{2 s_{1}} \frac{2\left(2 s_{1}-q\right)}{3 s_{1}} \cdot f(q) d q\right] \cdot \frac{1}{\mathrm{~N}} .
$$

Using the results of Section III and the equality

$$
\begin{aligned}
\int_{G}^{\infty} \exp (D u- & \left.u^{2} / 2\right) d u \\
& =\exp \left(D^{2} / 2\right) \cdot \int_{G-D}^{\infty} \exp \left(-u^{2} / 2\right) d u
\end{aligned}
$$

the expression (25) simplifies to

$$
\begin{aligned}
F\left(s_{1}\right)=\frac{1}{\bar{\gamma}}\left\{\bar{p} \cdot \Psi\left[s_{1}{ }^{(p)}-\sigma_{p}\right]-s_{1} \cdot \Psi \cdot\left[s_{1}{ }^{(p)}\right]+\right. \\
\\
\left.\quad 4 s_{1} \cdot \Psi\left[-2 s_{1}{ }^{(q)}\right]-2 \cdot \bar{q} \cdot \Psi\left[-2 s_{1}{ }^{(q)}+\sigma_{q}\right]\right\}
\end{aligned}
$$

where $\Psi\left[ \pm x^{(\alpha)} \pm y\right]$ is defined by (9).

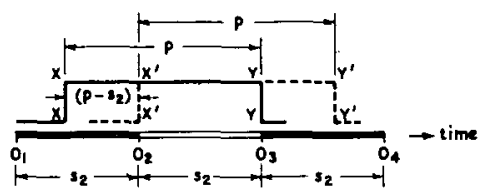

(a)

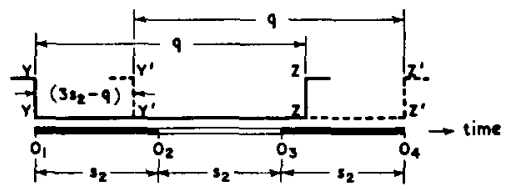

(b)

Fig. 2. Mechanism of transmission failure in $\mathrm{A} 2$. (a) $\mathrm{XX}^{\prime} / \mathrm{O}_{1} \mathrm{O}_{4}$ is the fraction of time for which the burst $\mathrm{XY}$ influences both the transmissions $\mathrm{O}_{1} \mathrm{O}_{2}$ and $\mathrm{O}_{3} \mathrm{O}_{4}$. (b) $Y Y^{\prime} / \mathrm{O}_{1} \mathrm{O}_{4}$ is the fraction of time for which adjacent bursts, separated by the interval $Y Z$, influence both the transmissions $\mathrm{O}_{1} \mathrm{O}_{2}$ and $\mathrm{O}_{3} \mathrm{O}_{4}$.

\section{Derivation of $F^{\prime}\left(s_{2}\right)$}

A failure can occur in scheme $\mathrm{A} 2$ if both the transmissions of a message block are influenced by a noise burst or by successive bursts. Let $s_{2}$ be the message block length. Referring to Fig. 2(a) and (b) and proceeding in the manner of the previous subsection, the following upper bound can be derived on the failure rate in the scheme.

$$
\begin{aligned}
F^{\prime}\left(s_{2}\right)=\frac{2}{3 \bar{r}}\left\{\bar{p} \cdot \Psi\left[s_{2}{ }^{(p)}-\sigma_{p}\right]-s_{2} \cdot \Psi\left[s_{2}{ }^{(p)}\right]+\right. \\
\left.\quad 3 s_{2} \cdot \Psi\left[-3 s_{2}{ }^{(q)}\right]-\bar{q} \cdot \Psi\left[-3 s_{2}{ }^{(q)}+\sigma_{q}\right]\right\} .
\end{aligned}
$$

\section{APPENDIX III}

\section{Derivation of $d_{1}$}

It is necessary to determine the value $d_{1}$, of $a_{1}$, which minimizes the probability sum

$$
\begin{array}{r}
P_{1}=\operatorname{Prob}\left(p>a_{1}\right)+\operatorname{Prob}\left(q<2 a_{1}\right) \\
=\frac{1}{\sqrt{2 \pi}} \int_{1 \sigma / \operatorname{pln}\left(a_{1} / p_{m}\right)}^{\infty} \exp \left(-u^{2} / 2\right) d u+ \\
\frac{1}{\sqrt{ } 2 \pi} \int_{-\infty}^{1 / \sigma_{q} \ln \left({ }^{2} a_{1} / q_{m}\right)} \exp \left(-u^{2} / 2\right) d u
\end{array}
$$

where the log-normal distributions of $p$ and $q$ have been reduced to the normal form (see Section III).

The value of $d_{1}$ is determined by the solution of $d P_{1} /$ $d a_{1}=0$. In other words, the slopes of the normal distribution function at the points $\left(1 / \sigma_{p}\right) \ln \left(d_{1} / p_{m}\right)$ and $\left(1 / \sigma_{q}\right) \ln$ $\left(2 d_{1} / q_{m}\right)$ should be equal and opposite. In view of the symmetry of the cumulative curve, this requires that

$$
\frac{1}{\sigma_{p}} \ln \left(d_{1} / p_{m}\right)=-\frac{1}{\sigma_{q}} \ln \left(2 d_{1} / q_{m}\right) .
$$

On simplification

$$
d_{1}=\left[p_{m}^{\sigma q} \cdot\left(q_{m} / 2\right)^{\sigma_{p}}\right]^{1 /\left(\sigma q+\sigma_{q}\right)} .
$$

The minimum value of $P_{1}$ is then

$$
P_{1_{M}}=2 \Psi\left[d_{1}^{(p)}\right] .
$$




\section{Derivation of $d_{2}$}

From arguments similar to those used in the derivation of $d_{1}$

$$
d_{2}=\left[p_{m}^{\sigma_{q}} \cdot\left(q_{m} / 3\right)^{\sigma_{p}}\right]^{1 /\left(\sigma_{p}+\sigma_{q}\right)} .
$$

and the minimum value of $P_{2}=$ Prob $\left(p>a_{2}\right)+$ Prob $\left(q<3 a_{2}\right)$ is

$$
P_{2 M}=2 \Psi\left[d_{2}{ }^{(p)}\right] \text {. }
$$

\section{Appendix IV}

\section{Derivation of $F_{B 1}$ and $F_{B 2}$}

Consider $N$ transmissions in scheme B1, each of length $3 d_{1}$. The total transmission time is $3 N d_{1}$ and includes, to a good approximation, $3 N d_{1} / \bar{\gamma}$ noise bursts and an equal number of burst-free intervals (for large $N, \bar{r}$ is the burst repetition period). At worst, each of these bursts and burst-free intervals can be associated with an independent probability $P_{1 M} / 2$ of corrupting a triplicate of bits (see Section IV-B and Appendix III). Therefore, the failure rate in the scheme has the upper bound

$$
\begin{aligned}
F_{B 1} & =\frac{3 N d_{1}}{\tilde{r}} \cdot 2 \cdot \frac{P_{1 M}}{2} \cdot \frac{1}{N} \\
& =\frac{6 d_{1}}{\tilde{r}} \cdot \Psi \cdot\left[d_{1}{ }^{(p)}\right] .
\end{aligned}
$$

From similar arguments, the upper bound on the failure rate in scheme $B 2$ reads

$$
F_{B 2}^{\prime}=\frac{4 d_{2}}{\bar{r}} \cdot \Psi\left[d_{2}{ }^{(p)}\right] .
$$

\section{ACKNowledgment}

The author thanks Prof. S. V. C. Aiya for suggesting the problem and for useful discussions, and the Council of Scientific and Industrial Research, New Delhi, for the award of a fellowship.

\section{References}

[1] S. V. C. Aiya, "Some characteristics of tropical thunderstorms," Nature, vol. 208, pp. 641-644, November 1965 .

[2] S. V. C. Aiya and K. N. Lakshminarayan, "Atmospheric radio noise bursts in the LF band at Bangalore," J. Res. NBS (Radio Science), vol. 69, sec. D, pp. 1351-1358, Óctober 1965.
[3] D. W. Hagelbarger, "Recurrent codes: easily mechanized burst-correcting binary codes," Bell Sys. Tech. J., vol. 38, pp. 969-984, July 1959.

[4] B. Reiffen, W. G. Schmidt, and H. L. Yudkin, "The design of an error-free data transmission system for telephone circuits," AIEE Trans. (Communication and Electronics), vol. 80, pp. 224-231, July 1961.

[5] M. Horstein, "Efficient communication through burst-error channels by means of error detection," IEEE Trans. on Communication Technology, vol. COM-14, pp. 117-126, April 1966.

[6] W. Lyons, "Error protection via time diversity," IEEE Trans. on Communication Technology (Correspondence), vol. COM-13, pp. 234-237, June 1965.

[7] N. S. Jayant, "An 'erasure' scheme for atmospheric noise burst interference," Proc. IEEE (Letters), pp. 1943-1944, vol. 54, December 1966 .

[8] K. N. Lakshminarayan, "Short term 'time' characteristies of impulsive atmospheric noise," $J$. Sci. Ind. Res. (India), vol. 21-D, pp. 228-233, July 1962 .

[9] A. B. Glenn, "Comparison of PSK vs FSK and PSK-AM vs FSK-AM binary coded transmission systems," IRE Trans. on Communications Systems, vol. CS-8, pp. 87-100, June 1960.

[10] S. N. Gupta, "Effect of receiver bandwidth on atmospherio radio noise bursts," Ph.D. dissertation, Indian Institute of Science, Bangalore, India, 1965.

[11] L. F. Fenton, "The sum of log-normal probability distributions in scatter transmission systems," IRE Trans. on Communications Systems, vol. CS-8, pp. 57-67, March 1960.

\section{Bibliography}

[1] W. Lyons, "Application of time diversity to multi-link data transmission," Proc. Radio Club Amer., vol. 41, December 1965.

[2] N. S. Jayant, "An erasure scheme for data transmissions corrupted by atmospherics," to be published.

N. S. Jayant was born in Bangalore, India, on January 9, 1946. He attended National College, Bangalore, and received the B.Sc. degree from Mysore University, Mysore, India, in 1962 and the B.E. degree from the Indian Institute of Science, Bangalore, in 1965, where he continued under Prof. S. V. C. Aiya as a Research Worker in the Department of Electrical Communication Engineering. He is presently working for his doctorate and is also conducting a course on Irformation Theory for post-graduate students at the department. He is a Research

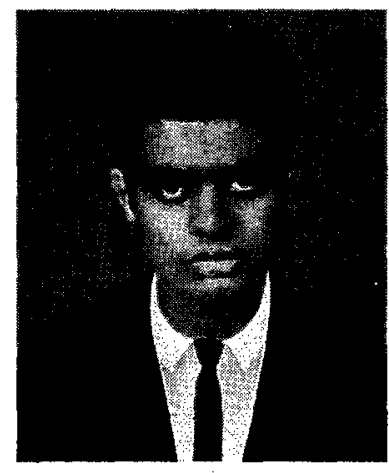
Fellow of the Council of Scientific and Industrial Research, New Delhi. 